\title{
Erratum to: First clinical results of minimally invasive vector lumbar interbody fusion (MIS-VLIF) in spondylodiscitis and concomitant osteoporosis: a technical note
}

\author{
Bernhard Rieger ${ }^{1,2} \cdot$ Hongzhen Jiang ${ }^{1,2,3}$ - Daniel Ruess ${ }^{4}$ Clemens Reinshagen ${ }^{5}$. \\ Marek Molcanyi $^{6,8} \cdot$ Jozef Zivcak $^{7}$ - Huaiyu Tong ${ }^{3}$ - Gabriele Schackert ${ }^{1,2}$
}

Published online: 17 February 2017

(c) Springer-Verlag Berlin Heidelberg 2017

\section{Erratum to: Eur Spine J}

DOI 10.1007/s00586-016-4928-z

In the original publication of the article, one of Marek Molcanyi's affiliations is missing.

Here is the detail of missing affiliation:

Department of Neurosurgery, Research Unit for Experimental Neurotraumatology, Medical University Graz, Graz, Austria.

The online version of the original article can be found under doi:10.1007/s00586-016-4928-z.

Bernhard Rieger

biokinemetrie@gmail.com

1 Department of Neurosurgery, Carl Gustav Carus University Hospital, Technical University of Dresden, 01307 Dresden, Germany

2 Spine Center DWG Level I, Carl Gustav Carus University Hospital, Technical University of Dresden, 01307 Dresden, Germany

3 Department of Neurosurgery, Chinese PLA General Hospital, Beijing 100853, China

4 Department of Stereotactic and Functional Neurosurgery, University of Cologne, 50937 Cologne, Germany

5 Department of Radiology, Massachusetts General Hospital, Harvard Medical School, Boston, MA, USA

6 Institute of Neurophysiology, Medical Faculty, University of Cologne, 50937 Cologne, Germany

7 Department of Biomedical Engineering, Technical University of Kosice, 04000 Kosice, Slovakia

8 Department of Neurosurgery, Research Unit for Experimental Neurotraumatology, Medical University Graz, Graz, Austria 